\title{
Study of behavioural risk factors for non- communicable diseases among adults in north Indian city population: Retrospective study
}

\author{
Sumit Lathwal
}

Assistant Director Health, Hq Delhi Area and Assistant Professor, Department of Community Medicine, Army College of Medical Sciences, New Delhi-110010, INDIA.

Email: sumitlathwal1@gmail.com

Abstract Background: Non communicable disease (NCD) include Hypertension, Type-II Diabetes Mellitus, Cardiac diseases, Obesity and Alcoholism have common risk factors in India and globally as well. Methodology: 84 patients aged between 35 and 65 having NCD were studied. Each patient was subjected to Routine blood examination, Haemoglobin estimation, $\mathrm{CBC}, \mathrm{RBS}, \mathrm{HbA}_{1} \mathrm{c}$, Lipid profile and Blood pressure was recorded with sphygnomanometer. Results: $21(25 \%)$ had obesity, 23 (27.3\%) had Type-2 DM, 17 (20.2\%) had Hypertension, 11 (13.0\%) were tobacco chewers and 12 (14.2\%) were alcoholic. In total cholesterol study (mg/dl) $23(27.3 \%)$ had 207.2 to 208.2, $29(34.5 \%)$ had 209.3 to 209.9 and 32 $(38.0 \%)$ had 210.3 to 212.2 levels. In HDL cholesterol study $(\mathrm{mg} / \mathrm{dl}) 29(34.5 \%)$ had 39.8 to $41.2,23(27.3 \%)$ had 42.9 to $44.8,32(38 \%)$ had 45.8 to 49.2 levels. In triglyceride (mg/dl) study $11(13 \%)$ had 95.2 to $99.2,32(38 \%)$ had 114.9 to $120.2,41(48.8 \%)$ had 136.5 to 148 . Conclusion: This pragmatic study will help to create awareness among general public and NCD patients about Obesity, HTN, DM and increased cholesterol levels and their risk factors so that risk of mortality and morbidity is reduced which is a quite common phenomenon in urban population.

Key Words: HTN = Hypertension, DM = Diabetes mellitus, NCD = Non communicable diseases, alcoholism, tobacco, sedentary life.

*Address for Correspondence:

Dr Sumit Lathwal, Assistant Professor, Department of Community Medicine, Room No 5/12, Delhi area single officers' accommodation, Cariappa Marg, Delhi Cantt, New Delhi-110010, INDIA.

Email: sumitlathwal1@gmail.com

Received Date: 05/12/2019 Revised Date: 12/01/2020 Accepted Date: 03/02/2020

DOI: https://doi.org/10.26611/10111431

\begin{tabular}{|l|l|}
\hline \multicolumn{2}{|c|}{ Access this article online } \\
\hline Quick Response Code: & Website: \\
& www.medpulse.in \\
& \\
\hline
\end{tabular}

\section{INTRODUCTION}

Non Communicable diseases include Obesity, Cardiac diseases, Type-2 Diabetes Mellitus, Hypertension and Alcoholism etc. Death due to non-communicable diseases were 58 millions (60.5\%) globally in $2005^{1}$. In India death due to non-communicable diseases was $54 \%$ and $44 \%$ people could not lead normal healthy life due to being frequently admitted to hospital and also dependent on medicines $^{2}$. As these non communicable disease (NCD) have high risk of mortality and morbidity ${ }^{3}$ WHO has recommended certain protocols to keep such patients healthy ${ }^{4}$. The steps include collection of information on 1socio-demographic variables, behavioural risk factors like alcohol use, tobacco use, physical inactivity, diet and related factors; 2-obtaining measurements such as weight, height, waist circumference, blood pressure; 3-lipid profile, HDL cholesterol, blood glucose and triglycerides. By evaluating all three protocols of WHO we have studied the various Non-communicable Diseases (NCD) at different age and sex to evaluate the reason of NCD to create awareness, treatment and adequacy of control of type-II DM and HTN.

\section{MATERIAL AND METHODS}

84 patients aged between 35 and 65 years visiting a tertiary care hospital in North India were studied. 
Inclusive criteria: Alcoholism, tobacco chewing, Hypertension (HTN), Obesity Type-2 Diabetes Mellitus (DM-2) were selected for study.

Exclusion Criteria: Patients with Pulmonary Tuberculosis, Skin diseases like Scabies etc, HIV, Viral Fever, Hepatitis A/B were excluded from the study.

Method: Every patient's history was recorded. Blood examination - Routine $\mathrm{CBC}, \mathrm{Hb} \%$, RBS, $\mathrm{HbA}_{1} \mathrm{C}$, Lipid profile in obese, Blood pressure was recorded. Their physical activity, habits were also noted. The duration of study was Mar 2016 to Feb 2017.

Statistical analysis: The various non-communicable diseases were classified with percentages. The statistical analysis was done with SPSS 2007 software. The ratio of male and female was 2:1.

Ethical clearance taken from relevant Ethical Committee.

\section{OBSERVATION AND RESULTS}

Table-1 Clinical manifestations of non-communicable diseases- $21(25 \%)$ were obese, $23(27.3 \%)$ had type-2 DM, $17(20.2 \%)$ had HTN, $11(13 \%)$ were tobacco or Gutka chewers and 12 (14.2\%) were alcoholic

Table-2 Study of blood pressure in Hypertensive (HTN) patients- $6(35.2 \%)$ had systolic BP 130.2 and Diastolic BP 79.5, 9 (52.9\%) had systolic 131.1 and Diastolic 80.5, 2 (11.7\%) had systolic 292.2 and Diastolic 81.5 levels.

Table-3 Study of blood glucose in type-2 DM patients -5 $(21.7 \%)$ had 85.9 to $91.2,11(47.8 \%)$ had 92.8 to $97.1,7$ (30.4\%) had 98.2 to 111.2

Table-4 Study of lipid profile in non-communicable diseases- In the study of total cholesterol $23(27.3 \%)$ had 207.2 to $208.2,29(34.5 \%)$ had 209.3 to $209.9,32(38 \%)$ had 210.3 to 212.2 levels. In HDL cholesterol - 29 (34.5\%) had 39.8 to $41.2,23(27.3 \%)$ had 42.9 to $44.8,32(38 \%)$ had 45.8 to 49.2

In Triglyceride study - 11 (13\%) had 95.2 to $99.2,32$ (38\%) had 114.9 to 120.2 , $41(48.8 \%)$ had 136.5 to 148 levels.

Table 1: Clinical manifestation of Non-communicable diseases. (Total No. of Patients-84)

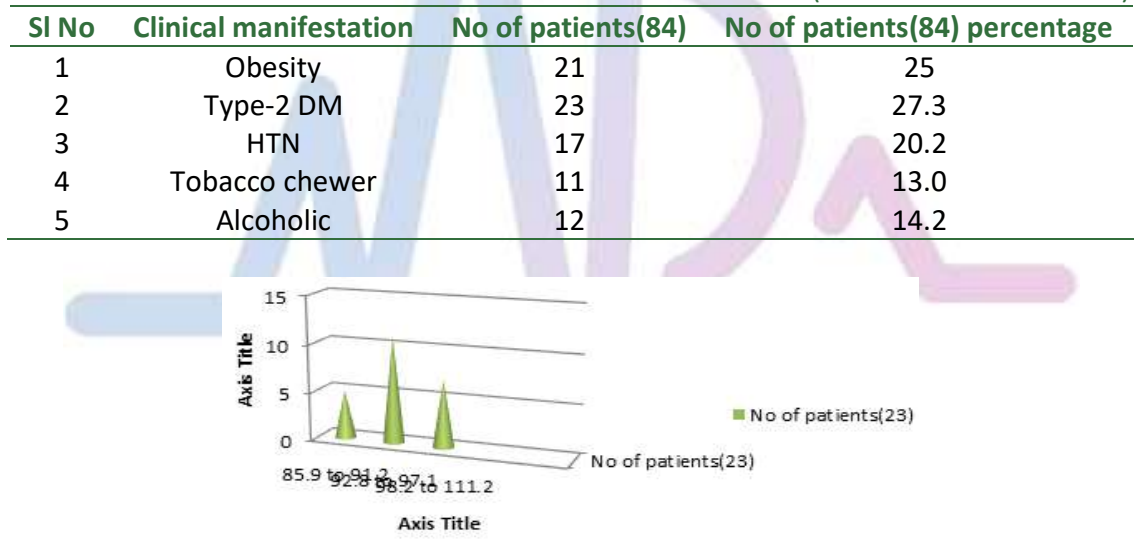

Figure 1: Clinical manifestation of Non-communicable diseases

Table 2: Study of blood pressure in HTN patients (Total No. of Patients-17)

\begin{tabular}{ccccc}
\hline \multirow{2}{*}{ SI No } & \multicolumn{2}{c}{ HTN(Blood pressure) } & \multirow{2}{*}{ No of patients(17) } & No of patients(17) percentage \\
\cline { 2 - 3 } & Systolic (mmHg) & Diastolic (mmHg) & & 35.2 \\
2 & 130.2 & 79.5 & 6 & 52.9 \\
3 & 131.1 & 80.5 & 9 & 11.7 \\
\hline
\end{tabular}

Table 3: Study of blood glucose in type-2 DM patients, No of patients (Total No. of Patients-23)

\begin{tabular}{cccc}
\hline SI No & Particulars & No of patients(23) & No of patients(23) percentage \\
\hline 1 & 85.9 to 91.2 & 5 & 21.7 \\
2 & 92.8 to 97.1 & 11 & 47.8 \\
3 & 98.2 to 111.2 & 7 & 30.4 \\
\hline
\end{tabular}




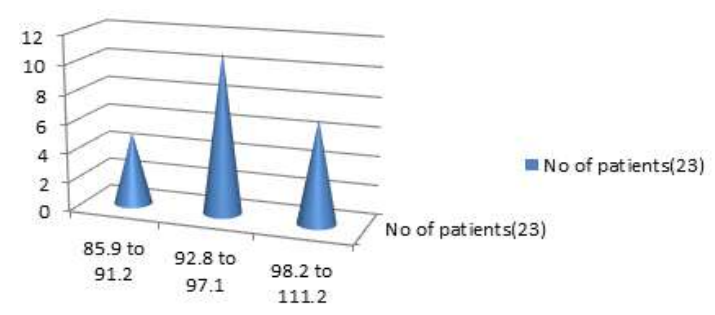

Figure 3: Study of blood glucose in type-2 DM patients

Table 4: Study of lipid profile

\begin{tabular}{cccc}
\hline 1 & Total cholesterol $(\mathrm{mg} / \mathrm{dl})$ & No of patients(84) & No of patients(84) percentage \\
\hline a & 207.2 to 208.2 & 23 & 27.3 \\
b & 209.3 to 209.9 & 29 & 34.5 \\
c & 210.3 to 212.2 & 32 & 38.0 \\
$\mathbf{2}$ & HDL cholesterol $(\mathbf{m g} / \mathrm{dl})$ & No of patients(84) & No of patients(84) percentage \\
a & 39.8 to 41.2 & 29 & 34.5 \\
b & 42.9 to 44.8 & 23 & 27.3 \\
c & 45.8 to 49.2 & 32 & 38.0 \\
3 & Triglyceride & No of patients(84) & No of patients(84) percentage \\
a & 95.2 to 99.2 & 11 & 13.0 \\
b & 114.9 to 120.2 & 32 & 38.0 \\
c & 136.5 to 148 & 41 & 48.8 \\
\hline
\end{tabular}

\section{DISCUSSION}

In the present study Non-communicable diseases among adult population of North Indian city was studied. 21 (25\%) were obese, 23 (27.3\%) had type-2 DM, 17 (20.2\%) had HTN, 11 (13\%) were tobacco chewers and $12(14.2 \%)$ were alcoholics (Table.1). The study of Blood pressure was among $17(20.2 \%)-6(35.2 \%)$ had $130.2 \mathrm{mmHg}$ systolic and $79.5(\mathrm{mmHg})$ diastolic, $9(52.9 \%)$ had 131.1(mmHg) systolic and 80.5 diastolic $(\mathrm{mmHg}), 2$ $(11.7 \%)$ had $129.2(\mathrm{mmHg})$ systolic and 81.5 diastolic (mmHg) (Table 2). In the study of blood glucose in type-2 DM 23 patients $(27.3 \%)-5(21.7 \%)$ had 85.9 to $91.2,11$ ( $47.8 \%)$ had 92.8 to $97.1,7$ (30.4\%) had 98.2 to 111.2 (Table-3). In the study of lipid profile - In total cholesterol study of (84 patients)- 23 (27.3\%) had 207.2 to 208.2 (mg/dl), 29 (34.5\%) had 209.3 to $209.9(\mathrm{mg} / \mathrm{dl}), 32(38 \%)$ had 210.3 to $212.2(\mathrm{mg} / \mathrm{dl})$. In HDL cholesterol $(\mathrm{mg} / \mathrm{dl}) 29$ $(34.5 \%)$ had 39.8 to $41.2(\mathrm{mg} / \mathrm{dl}), 23(27.3 \%)$ had 42.9 to $44.8(\mathrm{mg} / \mathrm{dl}) 32(38 \%)$ had 45.8 to $49.2(\mathrm{mg} / \mathrm{dl})$. In triglyceride (mg/dl) $11(13 \%)$ had 95.2 to $99.2(\mathrm{mg} / \mathrm{dl}), 32$ (38\%) had 114.9 to 120.2 , $41(48.8 \%)$ had 136.5 to 148 $(\mathrm{mg} / \mathrm{dl})$ (table.4). These findings are more or less in agreement with previous studies $5,6,7$. It was reported that, due to risk factors of NCD about 4.83 million premature deaths occurs globally. Long term abuse of alcohol may directly cause cirrhosis of liver, cancer of mouth, pharynx, GIT and increased risk of suicide and accident ${ }^{8,9}$. Tobacco chewing leads to fibrosis of oral cavity, malignancy of upper part of GIT and Peptic ulcers too. Inactivity or sedentary life leads to obesity which leads to hyper lipidemia/dyslipidemia to Ischemic heart diseases (IHD),
Myocardial infarction (MI) and Chronic Heart Diseases $(\mathrm{CHD})^{10}$. If obese population adapts to alcohol or tobacco chewing their inactivity or sedentary life will be enhanced and majority of sedentary patients are prone to Type-2 DM which affects micro vasculature and mainly affects cardio vascular system (CVS). ${ }^{11}$ In Type-2 DM due to hyper glycaemia, viscosity of blood is increased and rate of flow of blood is retarded (decreased) which causes peripheral vasculitis or cardiac vessel vasculitis which may cause sudden death also. In many patients poor blood flow causes ischemia, gangrene, optic neuritis due to blockage of central artery of retina which may cause permanent blindness, lack of sleep, Dementia, paraesthesia are also major symptoms of DM. DM may lead to social withdrawal and loneliness, anxiety and depression also. Hence, NCD includes HTN, hypercholestremia, stress, obesity, Type-2 DM, Cardiac Diseases, which ultimately leads to high mortality and morbidity. It is required to create awareness about the negative health effects of sedentary life, alcohol intake and tobacco chewing/smoking. If such individuals adopt healthy lifestyle including changing to healthy diet and exercising regularly it will lead to normal healthy life and Mortality and morbidity can be prevented/reduced.

\section{SUMMARY AND CONCLUSION}

The present study is on NCD's i.e. Obesity, Type-2 DM, HTN, Cardiac diseases and Alcoholism. A large scale awareness pogramme is required to educate the general public and also people suffering from Non-communicable diseases about these diseases and their risk factors. 
Moreover central and state governments must ensure strict legislation to stop completely or minimize alcohol and tobacco use.

\section{REFERENCES}

1. Strang $\mathrm{K}$, Mathers $\mathrm{C}-$ Preventing chronic diseases how many lives can we save? Lancet. 2005, $366: 1578-82$

2. Reddy KS Shah B - Responding to the threat of chronic diseases in India Lancet - 2005 366, 1744-9

3. Epping - Jordan J.E - Preventing chronic diseases; taking step wise action.Lancet 2005 366; 1667-71

4. Bontia R, de Courten M-Surveillance of risk factors for non-communicable diseases The WHO stepwise approach Geneva Switzerland. World Health Organization 2002

5. Srinath Reddy K - Cardio Vascular Diseases in the developing countries dimensions, determinates, dynamics and direction for public health action, public heath nutr.2002 5; 231.7

6. Salin Y Srinath R, Stephanie O - Global burden of CardioVascular diseases, Circulation 2001, 104. 2746-53

7. Gaffar A, Reddy K S - Burden of Non-Communicable diseases in South Asia BMJ. 2004, 328; 807-10

8. Park K, In : Park's text book of preventive and social medicine $15^{\text {th }}$ edition Jabalpur $\mathrm{M} / \mathrm{s}$ BanaroidsBahmot Publishers. 1997, 268-301

9. Coleman. CA, Friedman AG - The relationship of daily stress and health related behaviors of adolescent's cholesterol levels. Adolescence 1998,33,447-60

10. Kannan KP, Thankappan KR - Health and development in rural Kerala, Trivandrum, Kerala sastrasahityaparishad. 1991

11. Osuntokum $\mathrm{BO}-$ The changing patterns of diseases in developing countries World Health Forum. 1985, 6, 3103.

\section{Source of Support: None Declared \\ Conflict of Interest: None Declared}

Policy for Articles with Open Access:

Authors who publish with MedPulse International Journal of Community Medicine (Print ISSN: 2579-0862) (Online ISSN: 2636-4743) agree to the following terms: Authors retain copyright and grant the journal right of first publication with the work simultaneously licensed under a Creative Commons Attribution License that allows others to share the work with an acknowledgement of the work's authorship and initial publication in this journal.

Authors are permitted and encouraged to post links to their work online (e.g., in institutional repositories or on their website) prior to and during the submission process, as it can lead to productive exchanges, as well as earlier and greater citation of published work. 